\title{
Origin of photovoltage in perovskite solar cells probed by first-principles calculations
}

Cite as: Appl. Phys. Lett. 112, 233902 (2018); https://doi.org/10.1063/1.5033325

Submitted: 04 April 2018 . Accepted: 27 May 2018 . Published Online: 07 June 2018

C. Echeverría-Arrondo (iD)
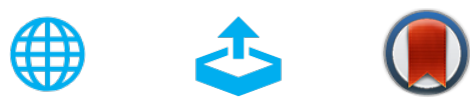

View Online

Export Citation

\section{ARTICLES YOU MAY BE INTERESTED IN}

Calcium doped MAPb/3 with better energy state alignment in perovskite solar cells

Applied Physics Letters 112, 193901 (2018); https://doi.org/10.1063/1.5020840

Flexible and air-stable perovskite network photodetectors based on $\mathrm{CH}_{3} \mathrm{NH}_{3} \mathrm{~Pb} \mathrm{P}_{3} / \mathrm{C} 8 \mathrm{BTBT}$ bulk heterojunction

Applied Physics Letters 112, 233301 (2018); https://doi.org/10.1063/1.5024330

Surface properties of lead-free halide double perovskites: Possible visible-light photocatalysts for water splitting

Applied Physics Letters 112, 243901 (2018); https://doi.org/10.1063/1.5035274

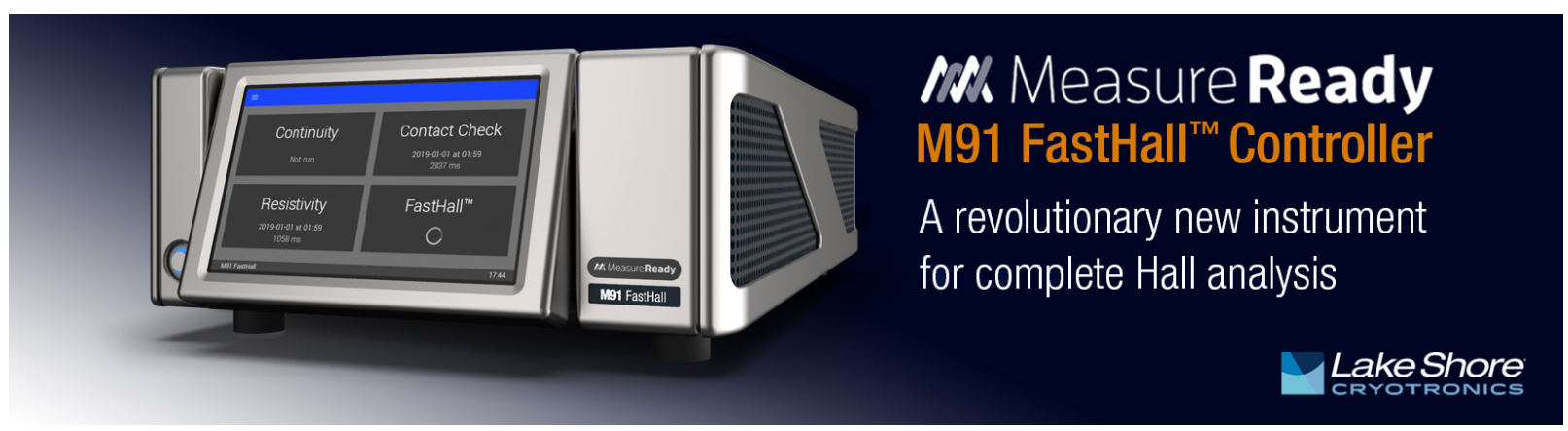




\title{
Origin of photovoltage in perovskite solar cells probed by first-principles calculations
}

\author{
C. Echeverría-Arrondo ${ }^{\text {a) }}$ \\ Institute of Advanced Materials (INAM), Universitat Jaume I, 12006 Castelló, Spain
}

(Received 4 April 2018; accepted 27 May 2018; published online 7 June 2018)

\begin{abstract}
Hybrid halide perovskite solar cells hold great potential for photovoltaic applications, but suffer, however, from anomalous current density-voltage characteristics. With a view to further understanding the performance of these optoelectronic devices, we investigate a prototypical electron selective contact with density functional theory methods. Our computations on a $\mathrm{TiO}_{2} /$ $\mathrm{CH}_{3} \mathrm{NH}_{3} \mathrm{PbI}_{3}$ heterojunction doped with Schottky defects at open circuit reveal a consistent picture of ions and interlayer excitons at the origin of photovoltage formation. Published by AIP Publishing. https://doi.org/10.1063/1.5033325
\end{abstract}

Solar cells based on perovskite semiconductors are at the forefront of photovoltaics research due to increasing power conversion efficiencies ${ }^{1-4}$ and lowering production costs. Large research effort is now centered on the long-term stability of the cell performance and the problem of dynamical hysteresis. ${ }^{5,6}$ This anomalous phenomenon is expressed as a deviation of the measured current from the classic Shockley ideal diode equation due to capacitance currents $^{7-11}$ arising from space charge layers formed at the contacts during the "past history" of the device. The capacitive response of hybrid lead halide solar cells has been largely probed, both in the $\operatorname{dark}^{7,10,11}$ and under illumination, ${ }^{8-10,12}$ with different techniques such as the open-circuit voltage decay and impedance spectroscopy. Under illumination, significant ion migration occurs toward the contacts, where a change in surface band bendings and concentrations of majority carriers results in an electrostatic contribution to the photovoltage. This enhancement is governed by the slow dynamics of cations out of the space charge layers and persists for a long period of seconds after illumination. ${ }^{12}$ High efficient solar cells, hence entail optimized selective contacts and null hysteretic effects on the $j-V$ curves.

With the goal of better understanding the role of ions and photogenerated carriers in the operation of perovskite solar cells, in this paper, we research an electron selective contact at the atomic level, $\mathrm{TiO}_{2} / \mathrm{CH}_{3} \mathrm{NH}_{3} \mathrm{PbI}_{3}$ (abbreviated as $\mathrm{MAPbI}_{3}$ ). The studied heterojunction belongs to a prototypical multilayer design: ${ }^{13}$ Glass/FTO/TiO $/ \mathrm{MAPbI}_{3} / \mathrm{Spiro}-$ $\mathrm{OMeTAD} / \mathrm{Au}$; here, FTO refers to fluorine-doped tin oxide (ITO is used as well) and Spiro-OMeTAD is an organic coating. In this stack, the perovskite photoactive layer is sandwiched between an electron collecting cathode $\left(\mathrm{TiO}_{2}\right.$ as the electron transport layer plus FTO as the oxide cathode) and a hole collecting anode (Spiro-OMeTAD as the hole conductor and $\mathrm{Au}$ as the metallic anode). The whole structure of the bilayer is depicted in Fig. 1.

The theoretical description of this system is based on density functional theory (DFT) calculations carried out with VASP. ${ }^{14-16}$ This code is based on the projector augmentedwave (PAW) method, characterized by smooth valence wave

a)cechevar@uji.es functions close to the frozen atomic cores. ${ }^{17}$ Computations are run on a single $k$ point, the $\Gamma$ point, with a cutoff energy of $460 \mathrm{eV}$, with PAW pseudopotentials generated with the generalized gradient approximation of Perdew-BurkeErnzerhof to the exchange-correlation functional, ${ }^{18}$ with a Coulomb term accounting for the strongly correlated Ti-3d electrons $[U=4.2 \mathrm{eV}$ (Ref. 19)], with spin-orbit interactions and with dipole corrections. The target slab is contained in an orthorhombic unit cell—known as the supercell in DFT formalism-infinitely replicated in space with periodic boundary conditions so as to reproduce the extended system. Starting from an initial guess structure with $\mathrm{CH}_{3} \mathrm{NH}_{3} \mathrm{PbI}_{3}^{+}$ $\left(\mathrm{MA}^{+}\right)$molecules randomly oriented, we relax the atomic positions until the residual forces on the individual nuclei are smaller than $0.1 \mathrm{eV} / \AA$.

The target structure is formed by two contacting layers, $\mathrm{TiO}_{2}$ and $\mathrm{MAPbI}_{3}$. The $\mathrm{TiO}_{2}$ substrate is built with optimized lattice parameters $(a=b=3.903 \AA$; $c=9.716 \AA)$ in the anatase phase and the $\mathrm{MAPbI}_{3}$ slice is grown on it in a pseudocubic phase; the contacting planes are (101) and (100), respectively. ${ }^{20-22}$ The total crystal contains 1095 atoms in an orthorhombic unit cell whose dimensions are $19.5 \times 31.4 \times 36.0 \AA^{3}$ and which includes $10 \AA$ of void space along the $z$ direction minimizing reciprocal interactions with neighboring slabs. ${ }^{20}$ For the $\mathrm{MAPbI}_{3}$ termination in touch with $\mathrm{TiO}_{2}$, we choose MAI. ${ }^{23-25}$ On the interface plane, $3 \times 5$ unit cells of $\mathrm{MAPbI}_{3}$ are grown on top of $5 \times 3$ unit cells of substrate $\mathrm{TiO}_{2}$, yielding a lattice mismatch of $\simeq 3 \%$ in the $x$ direction and $<1 \%$ in the $y$ direction when compared with the bulk. ${ }^{22}$ This biaxial strain does not significantly affect the calculated band structure, ${ }^{26}$ but in real cells, it is an important source of degradation due to an increased ion migration. ${ }^{27}$ Since the contact area under consideration is of $\sim 6 \mathrm{~nm}^{2}$ and real $\mathrm{MAPbI}_{3}$ layers are about $500 \mathrm{~nm}$ width, ${ }^{13}$ we are dealing with a volume in the $\mathrm{MAPbI}_{3}$ phase of $\sim 3 \times 10^{-18} \mathrm{~cm}^{3}$. Furthermore, $\mathrm{TiO}_{2}$ dangling bonds are saturated with pseudohydrogens of fractional charge: ${ }^{28,29} \mathrm{H}_{4 / 3}$ for titanium and $\mathrm{H}_{2 / 3}$ for oxygen; the $\mathrm{MAPbI}_{3}$ free wall does not give dangling bonds - the integrated electron charge at edge and bulk-like iodide sites is the same-, and it is not passivated therefore. As usual, the goal of passivation is the removal of any surface state which might appear within the 


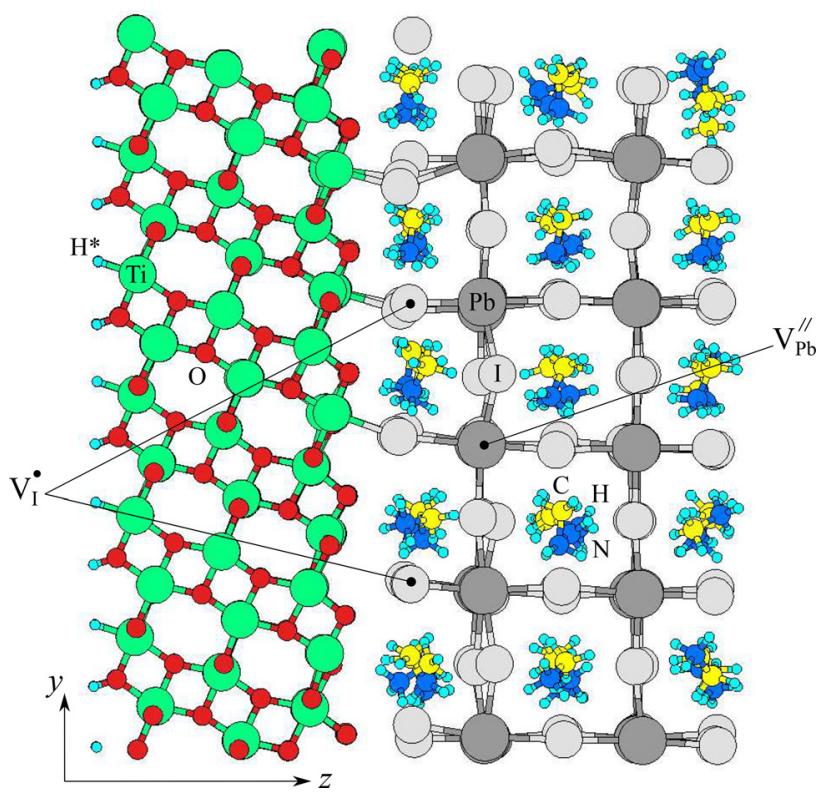

FIG. 1. Atomistic description of the $\mathrm{TiO}_{2} / \mathrm{MAPbI}_{3}$ heterojunction with the positions of $V_{\mathrm{I}}^{*}$ and $V_{\mathrm{Pb}}^{/ /}$ions; $\mathrm{H}^{*}$ stands for pseudohydrogens.

bandgap, as further confirmed by checking that the HOMO and LUMO states in the clean system are not contributed by atomic orbitals on the free wall. Moreover, passivation reduces the chemical reactivity of neighboring slabs.

Device processing introduces defects depending on growth conditions ${ }^{30}$ which are considered in the calculations for a realistic description of the heterointerface. The $\mathrm{TiO}_{2}$ slice contains $\mathrm{O}$ vacancies which provide free electrons to the conduction band (ionized donors) and its $n$-type character. Under $\mathrm{PbI}_{2}$ poor growth conditions, the $\mathrm{MAPbI}_{3}$ is of $p$ type due to $\mathrm{Pb}$ vacancies releasing free holes to the valence band (ionized acceptors). The dominant type of defects for perovskites are, nevertheless, Schottky pairs, ${ }^{31}$ which, in these growth conditions, form through the following reaction: ${ }^{31}$

$$
\oslash \rightarrow V_{\mathrm{Pb}}^{/ /}+2 V_{\mathrm{I}}^{\cdot}+\mathrm{PbI}_{2}
$$

where $\oslash$ represents the perfect $\mathrm{MAPbI}_{3}$ lattice and slashes and dots refer to negative and positive charges, respectively (Kröger-Vink notation). Schottky pairs appear in the lattice simultaneously and keep the charge neutrality. Under illumination, $V_{\mathrm{I}}^{*}$ and $V_{\mathrm{Pb}}^{/ /}$ions are pulled by the photovoltage towards the two opposite ends of the perovskite layer where they accumulate. Since we are accounting for a single Schottky pair and hence for three ions, the ionic density in a volume of $\sim 3 \times 10^{-18} \mathrm{~cm}^{3}$ is $\sim 10^{18} \mathrm{~cm}^{-3}$.

In dark conditions and open circuit, $V_{\mathrm{Pb}}^{/ /}$anions are dragged by the built-in field towards the selective contact. In order to investigate this situation, we proceed in two steps: First, we compute the system with one $V_{\mathrm{Pb}}$ vacancy, which yields two empty states by the valence band maximum [Fig. 2(a), left]. Second, we negatively charge $V_{\mathrm{Pb}}$ to $V_{\mathrm{Pb}}^{/ /}$[Fig. 2(a), center] and include two free holes on top of the valence band [Fig. 2(a), right]; these holes are provided by the abovementioned ionized acceptors and keep the system charge neutral. By holding these occupancies, we relax again the atomic positions in the system. The planar average of the (a) $\mathrm{V}_{\mathrm{Pb}}^{\prime \prime}$ anion
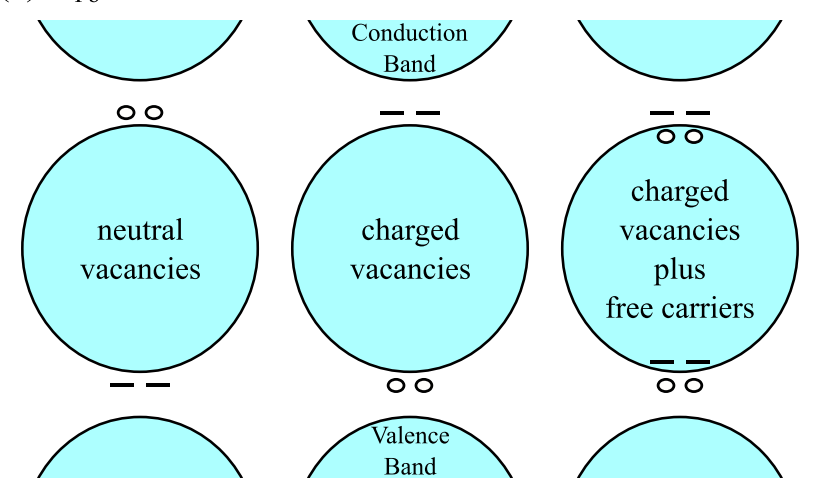

(b) $\mathrm{V}_{\mathrm{I}}^{\bullet}$ cations

FIG. 2. Schematic of bandgap regions showing the position of Schottky levels and free carriers for (a) one $V_{P b}^{/ /}$anion (dark) and (b) two $V_{I}^{*}$ cations (illumination).

electrostatic potential, ${ }^{32} \bar{V}(z)$ (Fig. 3, solid), gives the vacuum level, $E_{\text {vac }}=4.7 \mathrm{eV}$, and the $\mathrm{MAPbI}_{3}$ ionization energy, $I E=E_{\mathrm{vac}}-E_{\mathrm{VBM}} \sim 5.0 \mathrm{eV}[\simeq 5.3 \mathrm{eV}$ (Ref. 33)]. In order to subtract the microscopic oscillations from $\bar{V}(z)$, we integrate the macroscopic average (Fig. 3, dashed), which is a basic and useful concept in classical electromagnetism defined as ${ }^{32}$

$$
\overline{\bar{V}}(z)=(1 / a) \int_{z-a / 2}^{z+a / 2} \bar{V}\left(z^{\prime}\right) d z^{\prime} .
$$

Here, $a$ refers to the periodicity of $\bar{V}(z)$ on each side of the contact, namely, $a=3.7 \AA$ for $\mathrm{TiO}_{2}$ and $6.4 \AA$ for $\mathrm{MAPbI}_{3}$. The macroscopic average is found to jump from side to side of the contact, yielding a built-in voltage of $1.2 \mathrm{~V}$ in accord with the experiments. ${ }^{34}$ Computations provide as well the spreading of (Kohn-Sham) wave functions over the cross section (Fig. 4), and from them, we extract the relevant bandgaps and offsets: $1.6 \mathrm{eV}$ for $\mathrm{MAPbI}_{3}$, in consonance with optical experiments $; 35.7 \mathrm{eV}$ for $\mathrm{TiO}_{2}$, underestimated with respect to $3.2 \mathrm{eV}^{36}$ a total gap of $0.8 \mathrm{eV}$, close to the probed $\sim 1.1 \mathrm{~V} ; 33,37$ and an offset between valence band edges in $\mathrm{TiO}_{2}$ and $\mathrm{MAPbI}_{3}$ phases of $1.8 \mathrm{eV}[<2.2 \mathrm{eV}$ (Ref. 33)].

Under illumination, photocarriers create local potentials attracting the cations in the Schottky pairs towards the interface, where they contribute to enhance the photovoltage; ${ }^{6,12}$ the anions in the Schottky pairs are pushed toward the
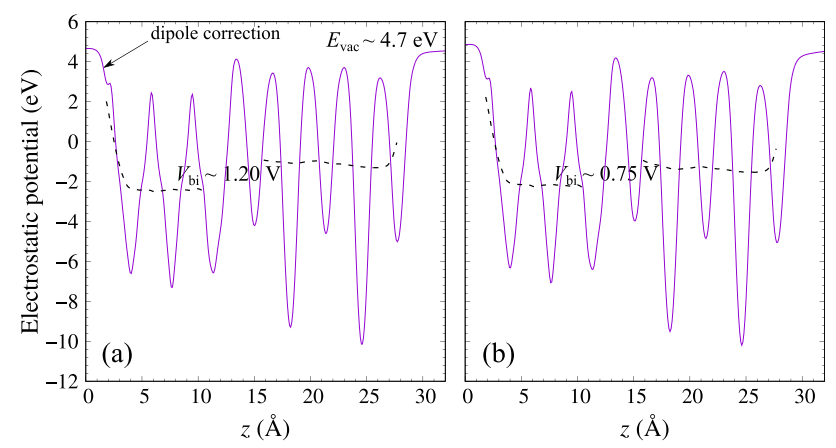

FIG. 3. Planar average (in solid) and macroscopic average (in dashed) of the electrostatic potential (a) in dark conditions and (b) under illumination. 
(a)
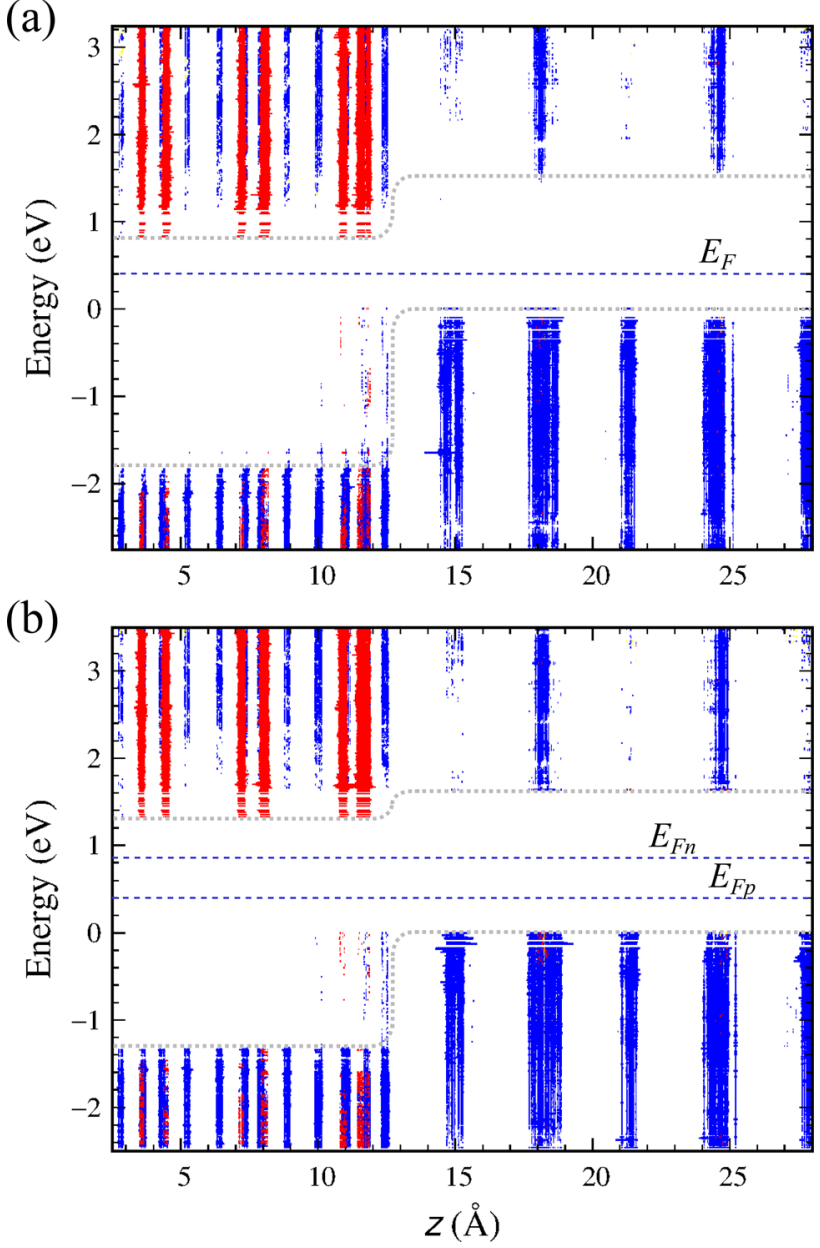

FIG. 4. Site projection of electron wave functions showing their spreading along the cross section and orbital character $(s, p$, or $d$ like as plotted in yellow, blue, and red, respectively). Panel (a) is for cells in the dark, and panel (b) is for cells under illumination. Fermi and quasi-Fermi levels are included for the sake of clarity; $E_{F}$ is half the total bandgap since donor and acceptor densities are assumed to be equal. The zero energy levels correspond to the valence band maxima, and gray dotted lines are drawn to guide the eye. Note the flattening of conduction and valence bands from dark to illumination conditions consistent with the formation of an $\sim 1 \mathrm{~V}$ photovoltage.

opposite contact, where they are expected to contribute similarly. ${ }^{33}$ To simulate this situation at open circuit, we proceed as in the dark case. First, we compute the system with two $V_{\mathrm{I}}$ vacancies, which yield two occupied states by the conduction band minimum [Fig. 2(b), left]. Second, we positively charge every $V_{\mathrm{I}}$ to $V_{I}^{*}$ [Fig. 2(b), center] and include two free electrons at the bottom of the conduction band [Fig. 2(b), right]; these electrons are provided by the abovementioned ionized donors. To reproduce the presence of photocarriers, we promote one valence electron to the conduction band and thereby create an electron-hole pair. Provided that the $\mathrm{MAPbI}_{3}$ volume is $\sim 3 \times 10^{-18} \mathrm{~cm}^{3}$, the density of photogenerated carriers is $\sim 3 \times 10^{17} \mathrm{~cm}^{-3}$, slightly larger than $4.2 \times 10^{16} \mathrm{~cm}^{-3}$ under one Sun illumination. ${ }^{38}$ By holding these occupancies-three electrons in the conduction band and three holes in the valence band-, we relax again the system. The spatially resolved spreading of wave functions along the cross section reveals a rise of band edges on the $\mathrm{TiO}_{2}$ side of $0.45 \mathrm{eV}$ between dark and illumination conditions (Fig. 4); this rise is consistent with an open-circuit photovoltage of $\sim 1 \mathrm{~V}^{33}$ and the same rise as obtained in the macroscopic average (Fig. 3). Although the

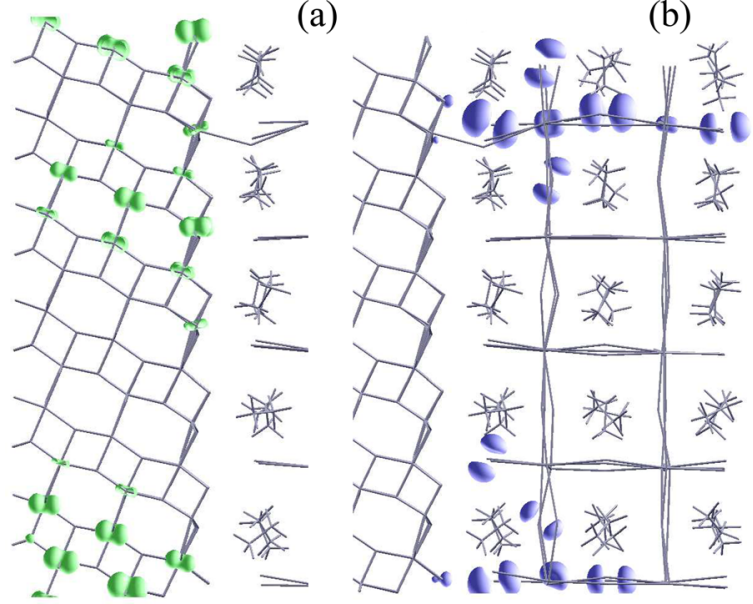

FIG. 5. Isosurfaces of constant charge density showing the spatial extension and location of the photocreated electron-hole pair on both sides of the contact. Panel (a) is for the bound electron and panel (b) is for the bound hole.

hole selective contact in this solar cell is not explicitly studied here, a flattening of $0.65 \mathrm{eV}$ is expected to occur based on Ref. 33, between the bands of $\mathrm{MAPbI}_{3}$ and Spiro-OMeTAD. Moreover, the clouds of sputtered dots on the left-hand sides of the frontiers in Figs. 4(a) and 4(b) also are illustrative: they show that valence states close to the valence band maximum are hybridized from $\mathrm{TiO}_{2}$ and $\mathrm{MAPbI}_{3}$ atomic orbitals; thus, the two layers under study are not separate systems but contacting. Figure 4 shows in addition that the conduction band minimum is placed on the $\mathrm{TiO}_{2}$ side and the valence band maximum is located on the $\mathrm{MAPbI}_{3}$ side. Photocarriers hence form interlayer excitons, as further confirmed by looking at the spatial distribution of their wave functions (Fig. 5).

In summary, in this work, we probe the formation of open-circuit photovoltage in a hybrid perovskite solar cell by first principles calculations carried out on a prototypical electron selective contact, $\mathrm{TiO}_{2} / \mathrm{CH}_{3} \mathrm{NH}_{3} \mathrm{PbI}_{3}$, doped with Schottky defects. Our results reveal that photovoltage is mainly originated at the contacts from the accumulation and the interplay between mobile ions and interlayer excitons. Based on these results, further research is suggested: on the $\mathrm{MAPbI}_{3} /$ Spiro-OMeTAD hole selective contact and on other prototypical solar cell configurations such as the inverted Glass/FTO/PEDOT:PSS/MAPbI 3 /PCBM/Al.

We thank C. Quarti and F. De Angelis for their help on the DFT calculations, N. López for the comments on the chemical reactivity of free surfaces, and J. Bisquert, S. Ravishankar, and A. Bou for their expertise on perovskite solar cells.

${ }^{1}$ H. Tang, S. He, and C. Peng, Nanoscale Res. Lett. 12, 410 (2017).

${ }^{2}$ A. Kojima, K. Teshima, Y. Shirai, and T. Miyasaka, J. Am. Chem. Soc. 131, 6050 (2009).

${ }^{3}$ N. G. Park, M. Gratzel, and T. Miyasaka, Organic-Inorganic Halide Perovskite Photovoltaics: From Fundamentals to Device Architectures, 1st ed. (Springer, Cham, Switzerland, 2016), p. VIII.

${ }^{4}$ M. M. Lee, J. Teuscher, T. Miyasaka, T. Murakami, and H. J. Snaith, Science 338, 643 (2012).

${ }^{5}$ S. Ravishankar, O. Almora, C. Echeverría-Arrondo, E. Ghahremanirad, C. Aranda, A. Guerrero, F. Fabregat-Santiago, A. Zaban, G. GarciaBelmonte, and J. Bisquert, J. Phys. Chem. Lett. 8, 915 (2017). 
${ }^{6}$ V. W. Bergmann, Y. Guo, H. Tanaka, I. M. Hermes, D. Li, A. Klasen, S. A. Bretschneider, E. Nakamura, R. Berger, and S. A. L. Weber, Appl. Mater. Interfaces 8, 19402 (2016).

${ }^{7}$ O. Almora, I. Zarazua, E. Mas-Marza, I. Mora-Sero, J. Bisquert, and G. García-Belmonte, J. Phys. Chem. Lett. 6, 1645 (2015).

${ }^{8}$ E. J. Juarez-Perez, R. S. Sanchez, L. Badia, G. Garcia-Belmonte, Y. S. Kang, I. Mora-Sero, and J. Bisquert, J. Phys. Chem. Lett. 5, 2390 (2014).

${ }^{9}$ I. Zarazua, J. Bisquert, and G. Garcia-Belmonte, J. Phys. Chem. Lett. 7, 525 (2016).

${ }^{10}$ A. Guerrero, G. García-Belmonte, I. Mora-Sero, J. Bisquert, Y. S. Kang, T. J. Jacobsson, J. P. Correa-Baena, and A. Hagfeldt, J. Phys. Chem. C 120, 8023 (2016).

${ }^{11}$ O. Almora, A. Guerrero, and G. García-Belmonte, Appl. Phys. Lett. 108, 043903 (2016).

${ }^{12}$ R. Gottesman, P. López-Varo, L. Gouda, J. A. Jiménez-Tejada, J. Hu, S. Tirosh, A. Zaban, and J. Bisquert, Chem 1, 776 (2016).

${ }^{13}$ J. M. Ball, S. D. Stranks, M. T. Horantner, S. Huttner, W. Zhang, E. J. W. Crossland, I. Ramirez, M. Riede, M. B. Johnston, R. H. Friend, and H. J. Snaith, Energy Environ. Sci. 8, 602 (2015).

${ }^{14}$ G. Kresse and J. Hafner, Phys. Rev. B 47, 558 (1993).

${ }^{15}$ G. Kresse and J. Furthmuller, Phys. Rev. B 54, 11169 (1996).

${ }^{16}$ G. Kresse and D. Joubert, Phys. Rev. B 59, 1758 (1999).

${ }^{17}$ P. E. Blöchl, Phys. Rev. B 50, 17953 (1994).

${ }^{18}$ J. P. Perdew, K. Burke, and M. Ernzerhof, Phys. Rev. Lett. 77, 3865 (1996).

${ }^{19}$ B. J. Morgan and G. W. Watson, J. Phys. Chem. C 114, 2321 (2010).

${ }^{20}$ J. M. Azpiroz, E. Mosconi, J. Bisquert, and F. D. Angelis, Energy Environ. Sci. 8, 2118 (2015).

${ }^{21}$ K. P. Ong, T. W. Goh, Q. Xu, and A. Huan, J. Phys. Chem. A 119, 11033 (2015).
${ }^{22}$ C. C. Stoumpos, C. D. Malliakas, and M. G. Kanatzidis, Inorg. Chem. 52, 9019 (2013).

${ }^{23}$ C. Quarti, F. D. Angelis, and D. Beljonne, Chem. Mater. 29, 958 (2017).

${ }^{24}$ L. She, M. Liu, and D. Zhong, ACS Nano 10, 1126 (2016).

${ }^{25}$ R. Ohmann, L. K. Ono, H.-S. Kim, H. Lin, M. V. Lee, Y. Li, N.-G. Park, and Y. Qi, J. Am. Chem. Soc. 137, 16049 (2015).

${ }^{26}$ C. Grote and R. F. Berger, J. Phys. Chem. C 119, 22832 (2015).

${ }^{27}$ Z. Jingjing, D. Yehao, W. Haotong, Z. Xiaopeng, Y. Zhenhua, S. Yuchuan, J. E. Shield, and H. Jinsong, Sci. Adv. 3, eaao5616 (2017).

${ }^{28}$ J. Li and L.-W. Wang, Phys. Rev. B 72, 125325 (2005).

${ }^{29}$ P. M. Kowalski, B. Meyer, and D. Marx, Phys. Rev. B 79, 115410 (2009).

${ }^{30}$ Q. Wang, Y. Shao, H. Xie, L. Lyu, X. Liu, Y. Gao, and J. Huang, Appl. Phys. Lett. 105, 163508 (2014).

${ }^{31}$ A. Walsh, D. O. Scanlon, S. Chen, X. G. Gong, and S.-H. Wei, Angew. Chem. Int. Ed. 53, 1 (2014).

${ }^{32}$ A. Baldesreschi, S. Baroni, and R. Resta, Phys. Rev. Lett. 61, 734 (1988).

${ }^{33}$ J. R. Harwell, T. K. Baikie, I. D. Baikie, J. L. Payne, C. Ni, and J. T. S. Irvine, Phys. Chem. Chem. Phys. 18, 19738 (2016).

${ }^{34}$ F. Zhang, X. Yang, H. Wang, M. Cheng, J. Zhao, and L. Sun, ACS Appl. Mater. Interfaces 6, 16140 (2014).

${ }^{35}$ Y. Yamada, T. Nakamura, M. Endo, A. Wakamiya, and Y. Kanemitsu, Appl. Phys. Express 7, 032302 (2014).

${ }^{36}$ L. Kavan, M. Gratzel, S. E. Gilbert, C. Klemenz, and H. J. Scheel, J. Am. Chem. Soc. 118, 6716 (1996).

${ }^{37}$ C.-C. Chueh, C.-Z. Li, and A. K.-Y. Jen, Energy Environ. Sci. 8, 1160 (2015).

${ }^{38}$ A. A. Paraecattil, J. D. Jonghe-Risse, V. Pranculis, J. Teuscher, and J.-E. Moser, J. Phys. Chem. C 120, 19595 (2016). 